\title{
Bases de datos y archivos municipales andaluces
}

El renacimiento de la Archivística que se deja sentir en la década de los setenta, llega con retraso a los archivos municipales. Sin embargo, una vez iniciada la renovación y a estas alturas, los cambios en el ámbito de esta Administración pública, en cifras absolutas, pueden estimarse como más notables que en el resto, sin que esto quiera decir que, en particular, para muchos archivos municipales la situación de incuria siga siendo habitual.

198I es una fecha significativa para Andalucía con la puesta en marcha del primer "Plan de Organización, y descripción de archivos municipales" de la provincia de Sevilla ', otras provincias como Huelva², primero, y Cádiz, después, se han sumado con planes equivalentes. Casi veinte años después, las transformaciones, no siendo suficientes ni generalizadas, también para nuestra región pueden valorarse como espectaculares respecto del punto de partida que hemos tomado como referencia, a sabiendas de que antes de esa fecha el vacío archivístico, por así decirlo, que no documental, puede constatarse salvo los casos de algunos archivos de capital de provincia.

A partir de dos tipos de radiografías que, al superponerse, completan el diagnóstico, hoy contamos con una imagen bastante fiable de todos y cada uno de los casi ochocientos archivos municipales andaluces. Dichas radiografías nos las proporcionan dos bases de datos BíBARA ${ }^{3}$ y CEN$\mathrm{SARA}^{4}$ que elaboradas por archiveros del Archivo General de Andalucía acaban de ver la luz con ocasión del XIV Congreso Internacional de archivos celebrado en Sevilla, y han quedado incorporadas al sitio web de dicho centro (www.aga.juntaandalucia.es) para una actualización permanente.

BÍBARA que es una recopilación bibliográfica archivística que parte de 1978, además de una información temática y por autores, nos facilita situándonos, en el subsistema de archivos de la Administración Local, la entrada en el conjunto de los archivos municipales y de cada uno de los que lo componen, permitiéndonos una valoración consistente, tanto general como particularizada.

Del total de ayuntamientos andaluces, sólo de 159 archivos municipales existe bibliografía con un total de 412 títulos. Dichos títulos, a simple vista, nos aportan datos sobre la planificación descriptiva en cada caso y sobre el grado de posibilidades informativas de los respectivos fondos y colecciones documentales.

A partir de los títulos recopilados observamos la diversidad de situaciones con respecto a dicha planificación que van desde la oportuna y conveniente, que parte de lo general y llega a lo particular, hasta la que se limita a sólo catálogos o ediciones de documentos. Esta variedad de situaciones tiene, lógicamente, que ver con la presencia o ausencia de archiveros. En este segundo caso, los historiadores han tomado el relevo de aquéllos, dejando a un lado determinados criterios archivísticos, convenientes y necesarios.

También, a partir de la bibliografía que afecta al ámbito municipal, se entrevé la diferencia, a favor de los primeros, entre los archivos de las ocho capitales de provincia y el resto de los archivos municipales.

Por lo que respecta a estos últimos, de entre las ocho provincias hay situaciones dignas de destacar por cuanto suponen acciones generales, planificadas, tempranas, continuadas y acrecentadas. De entre todas sobresale Sevilla, siguiéndole en la misma línea Huelva y Cádiz. Las tres cuentan con sendas colecciones editoriales que regularmente han ido publicando los instrumentos de descripción elaborados. En las tres provincias son las Diputaciones Provinciales las que han apoyado y patrocinado sus respectivos planes a instancia de sus archiveros y los resultados están traducidos en las ediciones de sus inventarios (65 para Sevilla, 33 para Huelva, 6 para Cádiz).

Situándonos en un plano general, la evaluación que venimos haciendo queda respaldada por los datos cuantitativos extraídos de
BÍBARA. Por provincias la situación es la siguiente:

\begin{tabular}{lccc} 
Provincia & Título & $\begin{array}{c}\text { correspon- } \\
\text { dientes a }\end{array}$ & $\begin{array}{c}\text { archivos } \\
\text { municipales }\end{array}$ \\
\hline Almería & 10 & $\ldots$ & 5 \\
Cádiz & 48 & $\ldots$ & 18 \\
Córdoba & 23 & $\ldots$ & 7 \\
Granada & 26 & $\ldots$ & 6 \\
Huelva & 46 & $\ldots$ & 36 \\
Jaén & 24 & $\ldots$ & 12 \\
Málaga & 28 & $\ldots$ & 8 \\
Sevilla & 207 & $\ldots$ & 67
\end{tabular}

La segunda radiografía nos la facilita la segunda base de datos aludida, CENSARA, que, como hemos, dicho recoge y actualiza el censo de los archivos del Sistema andaluz, entre otros de cada uno de los municipales.

Vuelve a constatarse de forma general la misma impresión: la diferencia lógica entre los archivos de capital de provincia y el resto, lo que no impide a su vez la diversidad de situaciones entre los ocho primeros. $\mathrm{Ni}$ que decir tiene que además de la diferencia por lo que afecta a volúmenes documentales, es la presencia de profesionales, por su número y por su formación, la que determina en buena medida la diversidad por lo que se refiere a programación y contenidos archivísticos.

Hasta no hace más de veinte años los archivos municipales, como tantos otros, tenían un lastre significativo: su adscripción a sólo la dimensión histórica. Algunos de los andaluces, incluso más de uno de capital de provincia, siguen sin romper amarras, convirtiéndose esa dimensión en una traba que impide el buen funcionamiento de la propia red de archivos del ayuntamiento en cuestión y de su sistema de información.

Basta con comprobar la falta de regularidad de las transferencias documentales, desde los archivos de gestión o de oficina, detectada en las estadísticas obtenidas de la última actualización del censo /diciembre del 99/ comparadas con las de la primera versión, /diciembre 1996/5 , sobre lo que abundaremos. 
Este estado de cosas dificulta, en consecuencia, la plena aceptación del principio de respeto a la edades de los documentos trascendiendo negativamente en la fluidez de las redes de archivos municipales.

Actualmente la red de archivos en cada ayuntamiento se suele constreñir, limitándose unas veces a los archivos de oficina y al central o "general", que hace de central, intermedio e histórico y otras veces se reduce a los archivos de oficina y al histórico, bastante alejado de aquéllos, tanto en el tiempo como en el espacio.

Insisto en que hemos tratado, a partir de los datos publicados del primer censo del Sistema andaluz correspondientes a 1996 y a partir de las estadísticas facilitadas por la última actualización, correspondiente a 1999, incorporada a CENSARA, hacer una evaluación correspondiente a estos últimos cuatro años.

En 1996 se recogió información de un total de 763 ayuntamientos que, a partir de la situación reflejada en los cuestionarios ${ }^{6}$, nos atrevimos a clasificar como 126 archivos y 637 depósitos documentales. Atrevimiento que nos valió más de una crítica, pero del que no nos arrepentimos.

1996 Archivos

$\begin{array}{lrr}\text { Almería } & 7 & 96 \\ \text { Cádiz } & 9 & 33 \\ \text { Córdoba } & 5 & 70 \\ \text { Granada } & 5 & 162 \\ \text { Huelva } & 6 & 74 \\ \text { Jaén } & 8 & 88 \\ \text { Málaga } & 8 & 90 \\ \text { Sevilla } & 78 & 22\end{array}$

En 1999, la información afecta a esos 763 ayuntamientos para los que, manteniendo nuestra línea de apreciación, estimamos como 161 Archivos y 594 depósitos documentales, lo que supone un cambio considerable y favorable. La reducción de los segundos a favor del aumento de los primeros empieza satisfacer los deseos que formulamos cuatro años atrás.

\begin{tabular}{lcc}
1999 & Archivos & $\begin{array}{c}\text { Depósitos } \\
\text { documentales }\end{array}$ \\
\hline Almería & 9 & 94 \\
Cádiz & 12 & 30 \\
Córdoba & 6 & 69 \\
Granada & 7 & 160 \\
Huelva & 24 & 56 \\
Jaén & 14 & 82 \\
Málaga & 9 & 89 \\
Sevilla & 88 & 13
\end{tabular}

No hemos renunciado a nuestra postura, porque precisamente aquella apreciación determina y explica muchos resultados ofrecidos que quedan limitados y ceñidos a sólo los estimados como Archivos.

Es el caso de la información referida a las plantillas. En 1996 dichas plantillas alcanzaban un total de 115 personas de las que 65 correspondían a los ocho archivos de capital de provincia y sólo 50 a 47 archivos municipales de la provincia, a los que había que añadir los 4 archiveros de zona responsabilizados de 69 archivos municipales en la provincia de Sevilla que están adscritos a su Diputación Provincial.

Curiosamente a finales de 1999, el personal de los archivos de capital de provincia ha disminuido en 5, ascendiendo la cifra en los municipales de la provincia que pasan de 50 a 92, sin contar los 4 archiveros de zona de Sevilla con 82 archivos a su cargo y los 2 archiveros de zona creados en Huelva responsabilizados de un total de 8 archivos.

Ayer y hoy, entre el personal responsable de los archivos prima la figura del archiverobibliotecario, que en el mejor de los casos comparte dedicación cuando no se inclina por las bibliotecas, seguido de los ayudantes de archivo, de los auxiliares de archivo y de los archiveros de zona, amén de otras figuras menos reconocibles.

En la actualidad sólo hay II Facultativos o equivalentes en los archivos de los pueblos y nada más que 7 para los ocho archivos de capital de provincia, ni siquiera uno por cada capital. No hay constancia en ninguno de los archivos municipales de personal técnico de reprografía o de restauración.

Hay algo que conviene destacar en cuanto a los recursos humanos, la provisionalidad va dando paso a la estabilidad, aunque no en la medida deseada.

En cuanto al volumen de los fondos y colecciones, tratándose de ayuntamientos con una gestión administrativa que necesariamente trasciende al volumen documental, los ingresos ni con mucho son tan elevados como cabría esperar, lo que denota un estatismo que tiene que ver con la falta de regularización de las transferencias, como apuntamos antes. Este estatismo se hace más notable en los archivos de capital de provincia. El siguiente cuadro, referido sólo a los estimados Archivos, nos aproxima al volumen total en $\mathrm{m} / \mathrm{l}$ del Patrimonio documental andaluz en dichos centros, en 1996, que al compararlo con las cifras obtenidas en 1999 , nos permite contabilizar la escasez de los referidos ingresos y sentir preocupación por ese patrimonio documental de hoy que será histórico mañana.

Las cifras hablan por sí solas.

\begin{tabular}{|c|c|c|c|}
\hline 1996 & archivos capitales & archivos de la provincia & total ingresos \\
\hline Almería & $1322 \mathrm{~m} / \mathrm{l}$ & $1630 \mathrm{~m} / \mathrm{l}$ & $2952 \mathrm{~m} / \mathrm{l}$ \\
\hline Cádiz & $2500 \mathrm{~m} / \mathrm{l}$ & $8858 \mathrm{~m} / \mathrm{l}$ & $11.358 \mathrm{~m} / \mathrm{l}$ \\
\hline Córdoba & $1566 \mathrm{~m} / \mathrm{l}$ & $2845 \mathrm{~m} / \mathrm{l}$ & $4411 \mathrm{~m} / \mathrm{l}$ \\
\hline Granada & $2214 \mathrm{~m} / \mathrm{l}$ & $1825 \mathrm{~m} / \mathrm{l}$ & $4039 \mathrm{~m} / \mathrm{l}$ \\
\hline Huelva & $2015 \mathrm{~m} / \mathrm{l}$ & $1499 \mathrm{~m} / \mathrm{l}$ & $3514 \mathrm{~m} / \mathrm{l}$ \\
\hline Jaén & $2186 \mathrm{~m} / \mathrm{l}$ & $3575 \mathrm{~m} / \mathrm{l}$ & $5761 \mathrm{~m} / \mathrm{l}$ \\
\hline Málaga & $3500 \mathrm{~m} / \mathrm{l}$ & $4460 \mathrm{~m} / \mathrm{l}$ & $7960 \mathrm{~m} / \mathrm{l}$ \\
\hline \multirow[t]{2}{*}{ Sevilla } & $5645 \mathrm{~m} / \mathrm{l}$ & $7925 \mathrm{~m} / \mathrm{l}$ & $13.570 \mathrm{~m} / \mathrm{l}$ \\
\hline & $20.948 \mathrm{~m} / \mathrm{l}$ & $32.617 \mathrm{~m} / \mathrm{l}$ & $53.565 \mathrm{~m} / \mathrm{l}$ \\
\hline 1999 & archivos capitales & archivos de la provincia & total ingresos \\
\hline
\end{tabular}

\begin{tabular}{|c|c|}
\hline Totales de las & $1242 \mathrm{~m}$ \\
\hline
\end{tabular}


La cortedad de datos en 1996 referidos al equipamiento nos impide una evaluación de los cambios al llegar a 1999 que será ciertamente el punto de partida para las estimaciones venideras en este punto.

La conservación física sigue siendo razonablemente aceptable, mientras que la seguridad sigue teniendo que ver con la presencia o ausencia de archiveros. Aunque se hace constar afirmativamente, en más de un caso, que se hacen estudios de identificación y valoración, la falta de especificación de los mismos hace pensar que se han quedado en estudios de identificación para la descripción, que no para la selección con vistas a la eliminación.

Algo más, en estas consideraciones de carácter general. Ningún sitio web en 1996. En 1999, también escasos si nos referimos a los propios: La Línea de la Concepción, Andújar. Sin embargo la información de los archivos municipales empieza a incorporarse a los sitios webs de los ayuntamientos respectivos, como es el caso de Córdoba, o de las Diputaciones Provinciales, en el caso de provincias con Planes de archivos.

Es muy posible que algunos Ayuntamientos o algunos archiveros, al acercarse a esas dos bases de datos y recuperar los de su archivo, no se vean satisfechos con la imagen obtenida. Se me ocurre recurrir a un mensaje positivo que figuraba en el protector de pantalla de los ordenadores que, con las bases de datos aludidas, se ofrecía a los asistentes en el stand de la Consejería de Cultura con ocasión del XIV Congreso Internacional de Archivos:

\section{¿No te gusta la imagen de tu archivo?}

\section{Cámbiala! Tienes ocasión y obligación de hacerlo. La fecha será el 31 de diciembre de 2000.}

Antonia Heredia Herrera

Directora del Archivo General de Andalucía

\section{Notas}

I. HEREDIA HERRERA, A. El plan de organización y descripción de archivos municipales de Sevilla. Proyecto y resultados. Actas del II Encontro Nacional de arquivos municipais en Montemornovo, Lisboa, 1992. pp. 63-74.

2. REY DE LAS PEÑAS, R. Plan de ordenación de archivos municipales de la Excma. Diputación Provincial de Huelva. Actas del II Congreso Nacional de ANABAD. Boletín de ANABAD, XXV, n² 2-3, 1985, pp. 24I-244.

3. HEREDIA HERRERA, A., RAVINA MARTÍN, M. BÍBARA, bibliografía archivística andaluza (19782000), Sevilla, Consejería de Cultura, 2000.

4. CENSARA, es una base de datos que ha recogido la última actualización del Censo de archivos del Sistema andaluz (diciembre, 1999) y permitirá incorporar las sucesivas actualizaciones.

5. HEREDIA HERRERA, A. Censo del Sistema andaluz de archivos. Sevilla. Consejería de Cultura, Archivo General de Andalucía, 1997.

6. Cfr. Censo del Sistema andaluz ... pp. 10 y ss.

7. Al reproducir las estadísticas totales y por provincias hemos evitado aquí volver a insistir, quedándonos sólo con las cifras totales.

\section{La restauración de las pinturas murales del Instituto Vicente Espinel de Málaga en CD-ROM}

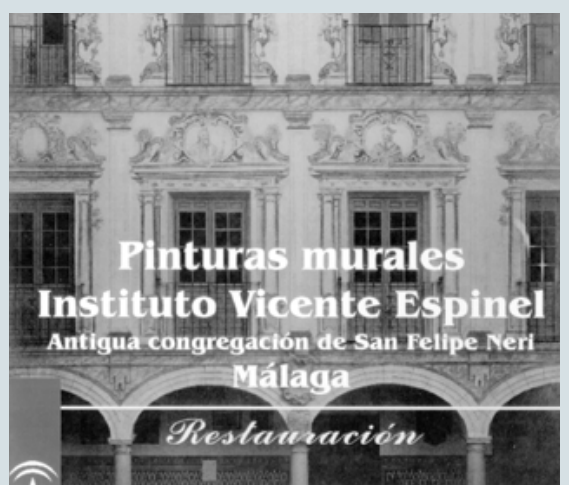

La restauración de las pinturas murales del Instituto Vicente Espinel, Antigua Congregación de San Felipe Neri, en Málaga, fue una obra coordinada por el Departamento de Bienes Muebles de la Dirección General de Bienes Culturales (Consejería de Cultura de la Junta de Andalucía) y realizada por la empresa Ágora, que quedó plasmada en este CD-ROM.

Este CD permite por una parte realizar un recorrido textual, y por otra ofrece la posibilidad de consultar la documentación visual o gráfica producida a raiz de la intervención.

Así, se pueden consultar los gráficos de los tratamientos, las fotografías realizadas previamente a la intervención, en el transcurso de ésta o aquéllas que reflejan el estado final, y los planos de todas las zonas restauradas.

En cuanto al recorrido textual, se inicia con una extensa Introducción en la que se explica el procedimiento seguido. Éste empezó con la redacción de un proyecto de conservación y restauración, con objeto de determinar el estado de conservación del conjun- to y establecer un tratamiento óptimo, para lo cual se analizaron los materiales, el estado de conservación y las patologías sufridas.

Asimismo, en este apartado se especifica el fin último de la intervención, unificar los espacios arquitectónicos del patio a partir de la decoración pictórica, y se justifica la inclusión, junto al estudio técnico, de una memoria histórica que complementa la visión del conjunto. Por último, al final de la introducción aparece la ficha técnica de la restauración realizada.

El siguiente botón nos permite acceder a la Memoria histórica, una muy extensa reseña en la que se hace referencia a la vida de Felipe Neri, la fundación en Italia de la congregación que lleva su nombre, su repercusión en España, y más tarde, la creación de la primera Congregación española de San Felipe Neri. 